\title{
Optoelectronic and material properties of nanocrystal-based CZTSe absorbers with Ag-alloying
}

\author{
Charles J. Hages, Mark J. Koeper, Rakesh Agrawal* \\ School of Chemical Engineering, Purdue University, 480 Stadium Mall Dr., West Lafayette, IN 47907, USA \\ E-mail addresses: chages@purdue.edu,mkoeper@purdue.edu, agrawalr@purdue.edu \\ *corresponding author: agrawalr@purdue.edu; Tel: +1 765494 2257; fax: +1 7654940805
}

Keywords: CZTSe, Ag-Alloying, ACZTSe, solution processing, nanoparticle, characterization

\begin{abstract}
In this work, the benefits of Ag-alloying in kesterite solar cells are explored in terms of tunable band gap, improved grain growth, improved minority carrier lifetime, reduced defect formation, and reduced potential fluctuations for $(\mathrm{Ag}, \mathrm{Cu})_{2} \mathrm{ZnSnSe}_{4}$ (ACZTSe) absorbers relative to $\mathrm{Cu}_{2} \mathrm{ZnSnSe}_{4}$ (CZTSe). The enhanced optoelectronic properties are shown to scale here with the degree of Ag-alloying in ACZTSe. The impacts of these effects on device performance are discussed, with improvement in average device performance/open-circuit voltage reported for ACZTSe (5\%-Ag) absorbers relative to CZTSe absorbers with similar band gap. These initial results are promising for the Ag-alloyed ACZTSe material system as $V_{O C}$ limitations are the primary cause of poor device performance in kesterite solar cells, and cation substitution presents a unique method to tune the defect properties of kesterite absorbers. Herein, nanoparticle synthesis and large-grain ACZTSe absorber formation is described followed by material and optoelectronic characterization. Additionally, RTP processing is presented to achieve fully selenized large-grain chalcogenide absorbers from sulfide nanocrystal inks.
\end{abstract}

\section{Introduction}

$\mathrm{Cu}_{2} \mathrm{ZnSn}(\mathrm{S}, \mathrm{Se})_{4}(\mathrm{CZTSSe})$ solar cells have gained recent research attention as lab-scale devices have achieved $12.6 \%$ power conversion efficiency (p.c.e.) [1]. However, CZTSSe performance is limited by low open-circuit voltages $\left(V_{O C}\right)$ which can be associated with the detrimental defect properties of the bulk absorber [2]. One route to improve device performance of CZTSSe absorbers is through material alloying, where various elements can be incorporated into the tetragonal crystal lattice to modify the optoelectronic properties of the absorber. Notably, Gealloyed $\mathrm{Cu}_{2} \mathrm{Zn}(\mathrm{Sn}, \mathrm{Ge})(\mathrm{S}, \mathrm{Se})_{4}(\mathrm{CZTGeSSe})$ has demonstrated improved performance for CZTSSe absorbers through improved optoelectronic properties and the ability for band gap tuning/grading of the film [3]. In this work, we further expand our research in kesterite-based alloy material systems to include $\mathrm{Ag}$-alloyed $(\mathrm{Ag}, \mathrm{Cu})_{2} \mathrm{ZnSnSe}{ }_{4}$ (ACZTSe), where modification of the absorber properties has similarly been achieved. Here we report the successful formation of ACZTSe solar cells from heterogeneous nanocrystal inks and characterize the role of Ag on the graingrowth, absorption, defect, and device properties of this material. Nominal atomic Ag-alloy concentrations of $[\mathrm{Ag}] /([\mathrm{Ag}]+[\mathrm{Cu}])$ at $0 \%, 5 \%$, and $50 \%$ are considered here for ACZTSe to understand the impact of significant Agalloying (i.e. 50\%-Ag) as well as low-level alloying/doping (i.e. 5\%-Ag) where similar band gaps for ACZTSe and CZTSe are achieved.

In the CZTSSe material system, significant defect formation is the result of low formation energies of charge-compensating defect clusters [4] - namely the high propensity for cation intermixing - aided by overlapping stable oxidation states of $\mathrm{Cu}^{+1,+2}, \mathrm{Zn}^{+2}$, and $\mathrm{Sn}^{+2,+4}$. Therefore, tuning the defect properties of CZTSSe absorbers may be possible through cation substitution in the kesterite lattice. Theoretical analysis has shown the main (compensating) defects which contribute to the free carrier properties of CZTSSe are the $\mathrm{Cu}_{\mathrm{Zn}}$ and $\mathrm{Zn}_{\mathrm{Cu}}$ antisite defects as well as $\mathrm{V}_{\mathrm{Cu}}$ vacancy [4], which all involve $\mathrm{Cu}$. Furthermore, these defect sites near the band edge are expected to contribute to detrimental potential fluctuations and donor-acceptor pair/free-to-bound recombination in the absorber [2,5-7]. Therefore, substitution of $\mathrm{Cu}$ with $\mathrm{Ag}$ in the ACZTSe alloy may allow for modification of the formation energy/density of these defects and/or their location in the energy gap of the absorber. In contrast, Gealloying is expected to modify deeper level recombination centers associated with the multivalent Sn element [3].

In addition to modification of the defect properties, Ag-alloying may also be beneficial for band gap tuning/grading of the absorber for improved performance. For CZTSSe, the absorber band gap $\left(E_{G}\right)$ is determined mainly by $\mathrm{Cu} d$ orbital and S/Se $p$ orbital anti-bonding (valance band maximum - VBM) and Sn $s$ orbital and S/Se $s p$ 
hybrid orbital anti-bonding (conduction band minimum - CBM) [8]. Therefore, $E_{G}$ tuning can be achieved through modification of the desired cation/anion interactions in the lattice. The most common technique for band gap tuning in CZTSSe is through S/Se substitution; for CZTS relative to CZTSe, the $p$ level is lower for $\mathrm{S}$ than Se, reducing the VBM, while the shorter bond length of $\mathrm{Sn}-\mathrm{S}$ relative to $\mathrm{Sn}-\mathrm{Se}$ increases the CBM through increased level repulsion of the anti-bonding state [8]. An alternative approach to band gap tuning is through $\mathrm{Sn} / \mathrm{Ge}$ alloying in CZTGeSSe; the shorter bond length of Ge-S/Se relative to Sn-S/Se increases the CBM due to an increase in the level repulsion for the anti-bonding state $[9,10]$. One benefit of the $\mathrm{Sn} / \mathrm{Ge}$-alloying for band gap control is that Ge leads solely to modification of the CBM, which can be beneficial to achieving a device structure with a back-surface field or band gap gradient for improved device performance.

However, it is also desirable to have an additional technique for tuning the VBM. The benefit of VBM level tuning is important when modification of $E_{G}$ is desired, however, a change in the CBM is not due to band alignment limitations at the $p n$-heterojunction interface. Additionally, device simulation has indicated a reduction in the VBM at the heterojunction interface can lead to an increase in device performance through improved opencircuit voltages [11]. To achieve VBM lowering, $\mathrm{Ag}$ can be alloyed with $\mathrm{Cu}$ to form $(\mathrm{Ag}, \mathrm{Cu})_{2} \mathrm{ZnSnS} \mathrm{S}_{4}(\mathrm{ACZTS})$, where $E_{G}$ of $\mathrm{Ag}_{2} \mathrm{ZnSnS}_{4}$ (AZTS) is $0.34 \mathrm{eV}$ greater than that of CZTS [10]. This $E_{G}$ increase is the expected result of a downshift in the VBM level from reduced group I-VI $p$ - $d$ hybridization; however, Ag-alloying may also result in a reduction of the $\mathrm{CBM}$ level due to anion position displacement, as reported for Ag-alloyed chalcopyrite semiconductors [12,13]. Therefore, a combination of group I, IV, and/or VI elements in the kesterite compound can be used to achieve the desired band structure.

Ag-alloying has been explored in the similar $\mathrm{Cu}(\mathrm{In}, \mathrm{Ga})(\mathrm{S}, \mathrm{Se})_{2}$ material system, where band gap tuning and modified defect properties have been identified for $(\mathrm{Ag}, \mathrm{Cu})(\mathrm{In}, \mathrm{Ga}) \mathrm{Se}_{2}$ (ACIGSe). Namely, modification of the absorber band gap and a decrease in potential fluctuations/free carrier density have been characterized with increasing Ag-content in the film [12,14]. Additionally, Ag-alloying in ACZTS has recently been reported, where (12 at.\%) Ag alloying has been associated with an increase in $E_{G}$, reduction in $V_{C u}$ defects, and a reduction in voids in the absorber [15]. Crystallographic and optical properties of ACZTS and ACZTSe have been reported [16], as well as thermoelectric properties of $\mathrm{Ag}_{2} \mathrm{ZnSnSe}_{4}$ (AZTSe) nanoparticles [17]. It should be noted that AZTS and AZTSe are reported with n-type semiconducting properties [17,18], which may be associated with a reduction in the p-type doping of these material due to Cu-related defects; therefore, AZTSe absorbers with greater than $50 \%-\mathrm{Ag}$ alloying were not considered for the present study.

\section{Experimental}

Nanoparticle synthesis described herein follows the procedures described by Miskin et al. [19] for CZTS synthesis. Additionally, Ag-incorporation is explored through substitution of silver acetylacetonate (98\% Sigma-Aldrich) in place of copper (II) acetylacetonate (97\% Sigma-Aldrich) in the nanoparticle synthesis, considered here with $[\mathrm{Ag}] /([\mathrm{Ag}]+[\mathrm{Cu}])=0.05$ and $[\mathrm{Ag}] /([\mathrm{Ag}]+[\mathrm{Cu}])=0.50$ loading in the reaction vessel. Energy-dispersive X-ray spectroscopy (EDX) analysis of the synthesized nanoparticles yields the ratio of group I:II:IV elements as 0.79:1.13:1 (CZTS), 0.84:1.12:1 $([\mathrm{Ag}] /([\mathrm{Ag}]+[\mathrm{Cu}])=0.05)$, and 0.71:1.38:1 $([\mathrm{Ag}] /([\mathrm{Ag}]+[\mathrm{Cu}])=0.52)$. While minimal elemental losses are typically characterized for the described nanocrystal-based absorbers following selenization [3,20], EDX analysis on the selenized film with 50 at. \% Ag loading was used to verify no notable changes in the atomic ratio exist following the heat treatment. Throughout the paper, the nominal at. $\% \mathrm{of} \mathrm{Ag}$ loading in the nanoparticle reaction is used to refer to the nanoparticles and selenized films following the notation: 5\%-ACZTS, 50\%-ACZTS, 5\%-ACZTSe, and 50\%-ACZTSe. Nanocrystal inks are coated on molybdenum (800 nm) coated soda-lime glass substrates.

In contrast to previously reported CZTSSe absorbers [19], CZTSe and ACZTSe fabricated here are selenized in a three zone rapid thermal processer (RTP) flow furnace. Selenium pellets are placed upstream of the coated nanocrystal films in an open graphite assembly (for thermal mass) and a flow of inert Ar gas is maintained to deliver a constant supply of Se vapor during processing. One benefit of RTP processing is the ability to deliver a constant supply of Se vapor to the film throughout the entire selenization process, resulting in fully selenized films with little to no residual sulfur in the crystal lattice (discussed in section 3.2). Absorbers fabricated here are selenized at $500^{\circ} \mathrm{C}$ (Se and substrate temperature) for $40 \mathrm{~min}$ - similar to that reported for tube furnace processing 
[19]; the sample and Se are rapidly heated with equivalent ramp rates in $<2$ min and cooled to $<250^{\circ} \mathrm{C}$ under a high flow of cool Ar gas in < $15 \mathrm{~min}$. Devices are completed as previously described [3] with CdS (25-50 nm): $\mathrm{ZnO}$ $(80 \mathrm{~nm}): \mathrm{ITO}(220 \mathrm{~nm}): \mathrm{Ni} / \mathrm{Al}$ Grids. However, no $\mathrm{MgF}_{2}$ anti-reflective coatings were considered for the devices reported here, in contrast to those previously reported $[3,19]$.

Scanning electron microscopy (SEM) and EDX data was taken on an FEI Quanta electron microscope. Crystallographic structure was analyzed by powder X-ray diffraction (XRD) using a Rigaku SmartLab diffractometer with $\mathrm{Cu} \mathrm{K}_{\alpha}$ (and minimal $\mathrm{K}_{\beta}$ ) radiation at $40 \mathrm{kV}$ and $40 \mathrm{~mA}$; grazing-incidence (GI-XRD) was also considered for certain nanoparticle samples to minimize signal from the substrate, with a grazing angle of $0.3^{\circ}$; absorbers were rotated at $10 \mathrm{rpm}$ during the measurement. Raman spectroscopy was taken at an excitation wavelength of $633 \mathrm{~nm}$ using a HORIBA-JY LabRam HR800 system.

Current-voltage (JV) measurements were taken under AM1.5 illumination on an Oriel Sol3A solar simulator with temperature control stage $\left(25^{\circ} \mathrm{C}\right)$, calibrated to $100 \mathrm{~mW} / \mathrm{cm}^{2}$ with an Oriel $91150 \mathrm{~V}$ silicon reference cell; 4point probe measurement techniques were utilized. AC external quantum efficiency (EQE) measurements were performed at $151 \mathrm{~Hz}$ using a lock-in amplifier and preamplifier for signal processing. $\mathrm{E}_{\mathrm{G}}$ estimation from $\mathrm{EQE}$ data follows that previously described [3] where dominant collection in the space charge region (W) is assumed according to $\mathrm{EQE} \approx 1-\exp [-\alpha \mathrm{W}]$ and a direct gap absorption coefficient $\alpha \propto\left(\mathrm{E}-\mathrm{E}_{\mathrm{G}}\right)^{1 / 2}$ [21,22]. Equivalent analysis results are obtained for $\mathrm{E}_{\mathrm{G}}$ and the characteristic Urbach tail energy $\left(E_{U}\right)$ utilizing $\mathrm{EQE}$ and internal quantum efficiency (IQE) data. Drive level capacitance profiling (DLCP) and capacitance-voltage (CV) measurements (taken at $25^{\circ} \mathrm{C}$ ) follow the procedures described by Heath et al. [23] with measurements taken at $2 \times 10^{5} \mathrm{~Hz}$ for $V_{A C}$ from 20-380 mV. The relative dielectric of CZTSSe and ACZTSe $(5 \% \mathrm{Ag})$ is taken to be $8.5 \varepsilon_{0}$ from previous admittance measurements of nanocrystal based CZTSSe [24]. Minimal light-soaking effects were observed in the capacitance response. Time-resolved photoluminescence (TRPL) measurements were performed using a $637 \mathrm{~nm}$ diode laser (pulse width <1 ns, $1 \mathrm{MHz}$ ) and an InGaAs photomultiplier tube (Hamamatsu H10330A-45) under low level injection conditions; the instrument response function was measured at $260 \mathrm{ps}$. To avoid the effect of degradation of the absorber layers in air [25], TRPL measurements were performed on absorbers with CdS deposited on top, as previously reported [3].

\section{Results \& Discussion}

\subsection{Nanoparticle Characterization}

XRD and Raman data for the synthesized 5\%-ACZTS and 50\%-ACZTS nanoparticles are presented in Fig. 1 in comparison to reference data. For 5\%-ACZTS, the measured data are similar to that reported for equivalently synthesized CZTS nanoparticles by Miskin et al. [19], as expected for this low degree of Ag incorporation. XRD data, Fig. 1(a), are in close agreement to reference tetragonal (I $\overline{4}$ ) CZTS [19], and Raman data, Fig. 1(b) are in close agreement to the expected modes for CZTS [26]. Shoulders near the main A1 mode of CZTS (c. $338.5 \mathrm{~cm}^{-1}$ ) in the Raman signal may be attributed to the presence of $\mathrm{Cu}_{2} \mathrm{SnS}_{3}$ (CTS) or off-stoichiometric CZTS nanoparticles which result from the heterogeneous nanoparticle synthesis recipe [19,27].

In contrast, the synthesized 50\%-ACZTS nanoparticles results in a multi-phase mixture, attributed here to $\mathrm{CZTS}$ and a $\mathrm{SnS}-\mathrm{Ag}_{2} \mathrm{~S}$ mixture. First, the main diffraction signal is correlated with the CZTS phase as identical peak locations are observed for the CZTS, 5\%-ACZTS, and 50\%-ACZTS nanoparticles; no Ag alloying into the main tetragonal phase is suggested as the peaks have no shift toward that of AZTS (as predicted by Vegard's Law [28] for a solid solution of CZTS/AZTS). However, shoulders on the low angle side of the main diffraction peaks as well as the sharp peak near $32^{\circ} 2$-Theta indicate the presence of a secondary phase (with relatively large grain size indicated by the reduced full-width-half-max). This secondary phase matches close to that of orthorhombic SnS (PNMA) (PDF: 01-073-1859); the presence of $\mathrm{SnS}$ is further verified from Raman analysis. However, XRD and Raman peaks for the $\mathrm{SnS}$ phase are slightly shifted from the reference data, which we attributed to Ag-incorporation into this phase as solid solutions of $\mathrm{Ag}_{2} \mathrm{~S}-\mathrm{SnS}$ have previously been reported [29]. The presence of such a solid solution results in the measured diffraction peaks also matching close to those of $\mathrm{Ag}_{8} \mathrm{SnS}_{6}$ (PDF 38-434) and $\mathrm{AgSnS}_{2}$ (PDF 38-245) (with slight modification of the lattice spacing). Furthermore, $\mathrm{Ag}_{2} \mathrm{~S}$ and $\mathrm{SnS}$ have common peaks in their 
Raman signal [30,31]. Therefore, further information regarding the degree of Ag-incorporation into this phase is necessary to determine the exact crystal structure.
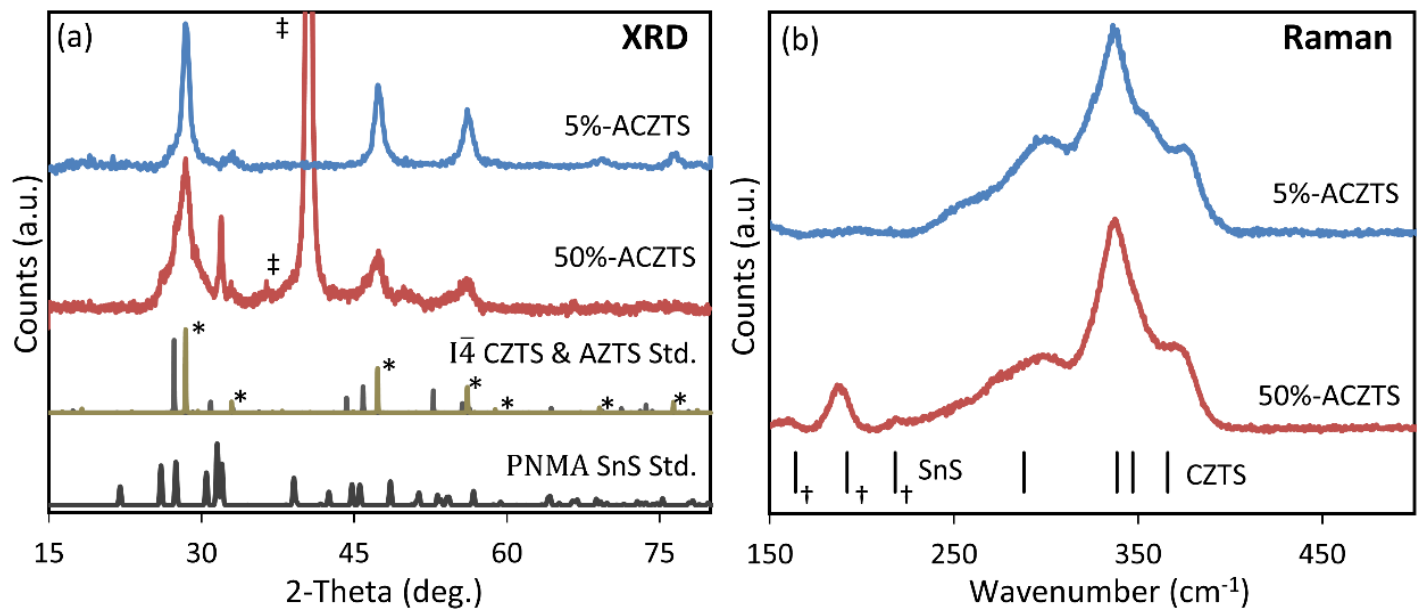

Fig. 1. (a) GI-XRD data for 5\%-ACZTS and XRD data for 50\%-ACZTS nanoparticles; ( $)$ indicates peaks related to the Mo (substrate) in the XRD measurement (not present in GI-XRD for 5\%-ACZTS); (*) indicates peaks associated with the CZTS standard. (b) Raman spectra comparison for the 5\%-ACZTS and 50\%-ACZTS nanoparticles; ( $\dagger$ ) indicates peaks related to $\mathrm{SnS}$ [30]. All other standard data for comparison is described in the text.

The shoulders near the main A1 mode of CZTS are relatively reduced for the 50\%-ACZTS nanoparticles, which we attribute to variations in composition and heterogeneity of the synthesized particles [27] related to the presence of Ag during nucleation and growth; particle size-separation following Carter et al. [27] indicates a reduction in small particle formation for the 50\%-ACZTS synthesis. Though the main Raman modes for CZTS are associated with the anion [26], modification of the cations (i.e. bonding) in the lattice are expected to result in shifting of the main Raman modes as similarly characterized for Ge-incorporation into CZTGeSSe [3]. Therefore, the lack of a shift or broadening for the 50\%-ACZTS Raman data associated with the tetragonal phase further verifies the lack of $\mathrm{Ag}$ in this phase and absence of an additional AZTS phase; all Ag measured through EDX analysis is expected in the (relatively large grain) $\mathrm{SnS}-\mathrm{Ag}_{2} \mathrm{~S}$ phase. A mass balance consideration suggests the existence of $\mathrm{ZnS}$ in the 50\%-ACZTS nanoparticles, though this phase is difficult to distinguish from XRD and nonresonant (w.r.t. ZnS) Raman analysis [20].

\subsection{Absorber Characterization}

Coated nanoparticle films of 5\%-ACZTS and 50\%-ACZTS were further processed into selenized thin-film absorbers through RTP processing; Raman and XRD data for the selenized films are presented in Fig. 2. Similar to the nanoparticle synthesis, XRD of 5\%-ACZTSe match close to that of tetragonal (II) CZTSe (PDF: 01-070-8930), shown in Fig. 2(a); Raman data also matches close to that reported for CZTSe [32], shown in Fig. 2(b). One significant difference in the selenized film data reported here relative to that previously reported from the Agrawal group is the absence of an additional peak near $320 \mathrm{~cm}^{-1}$ in the Raman data due to residual S in the crystal lattice [3] (for all RTP processed films). Additionally, XRD diffraction peaks do not indicate a shift in their peak location due to residual $\mathrm{S}$ in the crystal lattice found in previously reported devices [19]. The desire for sulfur-free films arises from the opportunity for reduced potential fluctuations compared to the S/Se alloy and elimination of detrimental electrical properties characterized for CZTSSe relative to CZTSe [33].

Despite the multi-phase nature of the 50\%-ACZTS nanoparticles, a single phase CZTSe/AZTSe alloy results for the 50\%-ACZTSe film following the selenization process. The formation of a single phase material from heterogeneous nanoparticles [27], as well as a mixture of binary and ternary nanoparticles [34], has previously been reported illustrating the high cation mobility during large-grain formation. Furthermore, uniform Ag incorporation from initially segregated electrodeposited metal layers during sulfurization has also been reported for ACZTS thin- 
films $[15,35]$. Therefore, the heterogeneous nanoparticles reported here are not expected to present a limitation to uniform ACZTSe formation.

XRD for the selenized 50\%-ACZTSe film shows a shift in the diffraction pattern to lower 2-theta relative to CZTSe. Furthermore, an increase in peak splitting for the main diffraction peaks, shown in the inset of Fig. 2(a) for the $\{220\}$ and $\{204\}$ peaks, is identified for 50\%-ACZTSe. These results illustrate an increase in lattice spacing for the ACZTSe films upon Ag-incorporation. Here we report the ACZTSe alloy with 50 at. \% nominal Ag alloying of tetragonal $(I \overline{4})$ structure with a/c $=0.516$; simulated $(I \overline{4})$ with the described increase in lattice spacing is plotted for comparison in Fig. 2(a), labeled "ACZTSe Std". This result is in close agreement with the expected a/c ratio for a 50\% CZTSe/AZTSe solid solution [28] (in comparison to $\mathrm{a} / \mathrm{c}=0.502$ for CZTSe and 0.534 for AZTSe [16]), as well as that previously reported for ACZTSe nanoparticles [16].
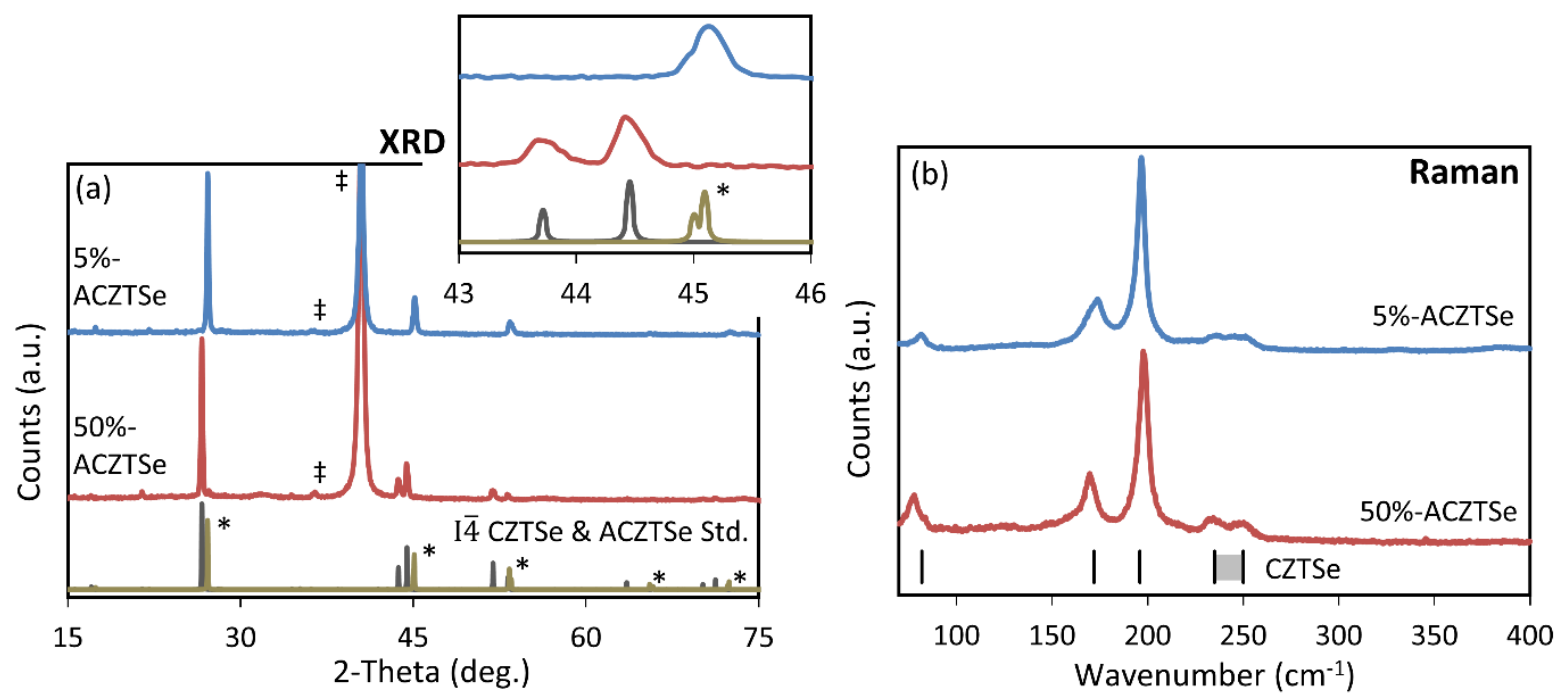

Fig. 2. (a) XRD data for the selenized 5\%-ACZTSe and 50\%-ACZTSe alloys; ( $\vdots$ ) indicates peaks related to the Mo (substrate); $(*)$ indicates peaks associated with the CZTSe standard; ACZTSe Std. is simulated $(I \overline{4})$ with $\mathrm{a} / \mathrm{c}=$ 0.516 ; the inset shows data near the $\{220\}$ and $\{204\}$ peaks. (b) Raman spectra comparison for the 5\%-ACZTSe and $50 \%$-ACZTSe alloys. All standard data for comparison is described in the text.

Raman spectroscopy of the 50\%-ACZTSe film shows similar data to that of 5\%-ACZTSe and CZTSe [32]. However, minor shifting of the main Raman modes are observed for 50\%-ACZTSe, with the main A and E symmetry modes identified at c. 78,170 , and $198 \mathrm{~cm}^{-1}$ in comparison to $82,174,197 \mathrm{~cm}^{-1}$, respectively, for $5 \%$ ACZTSe; minor B symmetry modes identified for CZTSe between $235-250 \mathrm{~cm}^{-1}$ [32] are also present in the signal, with minor variations in relative peak intensity characterized between the measured samples. Variations in the peak location and intensity characterized for 50\%-ACZTSe are attributed to modification of bonding in the crystal lattice due to Ag-incorporation, as similarly seen in Ge-alloyed CZTGeSSe absorbers [3], as well as variations in the short range order in the absorber [36], discussed further in section 3.3.

Cross-sectional and plain-view SEM images for the selenized ACZTSe absorbers, as well as a reference CZTSe absorber with equivalent processing, are shown in Fig. 3. Here we characterize notable changes in the grain size upon Ag-incorporation. All films demonstrate dense packing of the grains with no voids, as well as a bi-layer morphology which is commonly reported for selenized nanocrystal-based films [20]. However, a significantly larger average grain size with a reduction in the number of vertical grain boundaries is characterized for the ACZTSe films with increasing Ag-content relative to CZTSe - even with only 5 at. \% Ag alloying in the absorber. Additionally, $50 \%$-ACZTSe alloy is characterized with a reduction in the fine-grain layer thickness (located directly below the large-grain absorber). Typically, enhanced grain growth in CZTSe, as well as CIGSe absorbers, results from an increased liquid phase present in the film for liquid-assisted grain growth (such as $\mathrm{Na}-\mathrm{Se}, \mathrm{Cu}-\mathrm{Se}$, or other liquid phases [37-40]). Here, a low melting temperature $\left(217^{\circ} \mathrm{C}\right)$ eutectic for the $\mathrm{Cu}-\mathrm{Ag}-\mathrm{Sn}$ alloy (commonly used as a low temperature solder) may be responsible for enhanced liquid assisted grain growth and the increased grain size 
characterized for ACZTSe absorbers. Ag-incorporation in CIGSe has also been linked with reduced melting temperatures in this material system [12]. These results demonstrate promising initial results for improved grain growth of kesterite absorbers through Ag-alloying. Furthermore, only moderate concentrations of Ag present during absorber growth are required to achieve modification of the growth mechanism, as similarly found for alkali metal doping [37,41].
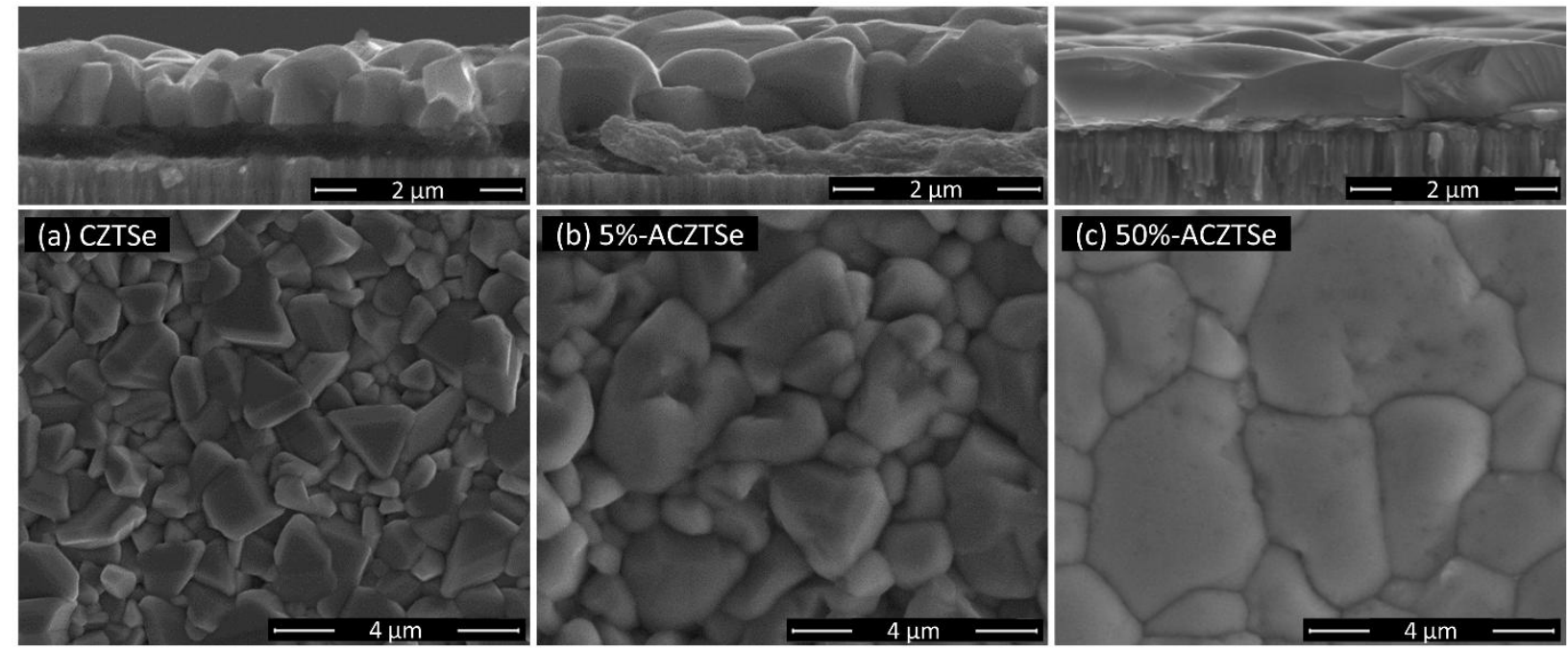

Fig. 3. Cross-sectional (top) and plain-view (bottom) SEM images for (a) CZTSe, (b) 5\%-ACZTSe, and (c) 50\%ACZTSe selenized absorbers.

\subsection{Optoelectronic Characterization}

TRPL analysis for the CZTSe and ACZTSe films was also considered, presented in Fig. 4(a). Here an increase in minority carrier lifetime is characterized for the ACZTSe absorbers with increasing Ag incorporation. For the 5\%ACZTSe alloy a 50\% increase in lifetime is characterized, while the 50\%-ACZTSe alloy demonstrates greater than $300 \%$ increase. These results are promising for kesterite absorbers as a low minority carrier lifetime is associated with performance limitations for CZTSSe devices $[2,42]$.
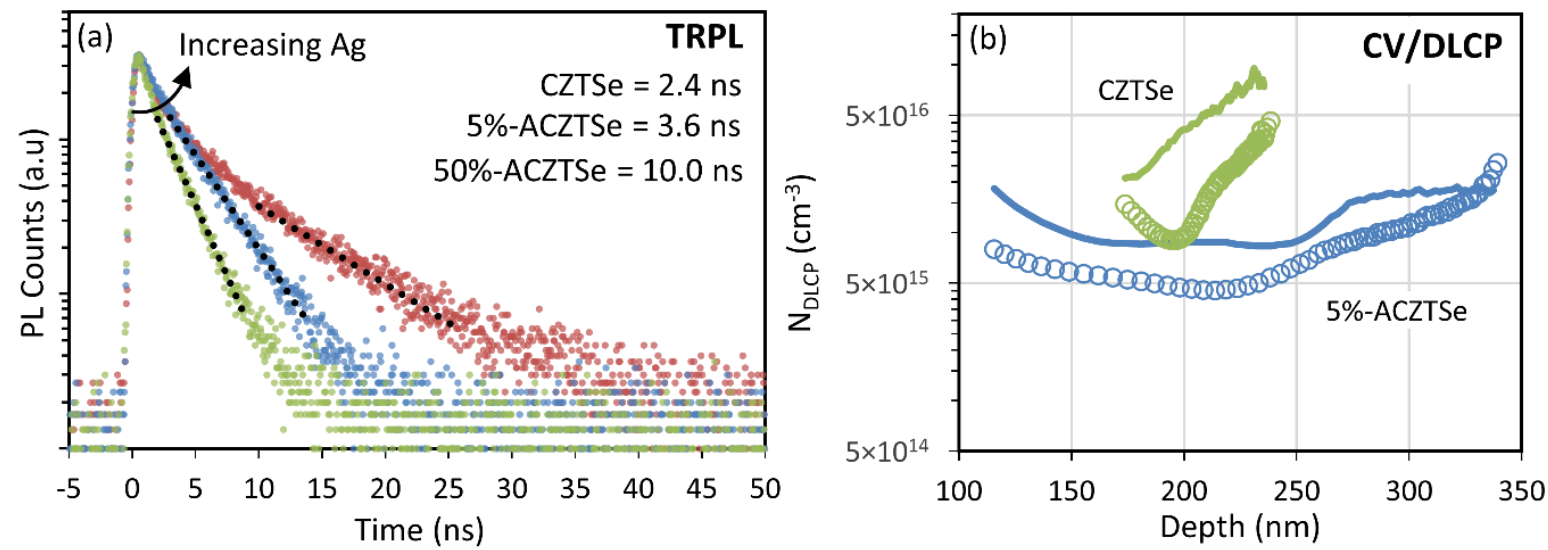

Fig. 4. (a) TRPL data for the CZTSe, 5\%-ACZTSe, and 50\%-ACZTSe selenized absorbers (with CdS); lifetime values reported are taken from an exponential fit to the TRPL data in the data range indicated on the plot (dashed lines). (b) CV (solid lines) and DLCP (data points) measured on representative CZTSe and 5\%-ACZTSe devices.

CV/DLCP data, presented in Fig. 4(b), also show a reduction in density and an increase in spatial uniformity of the free carrier density in 5\%-ACZTSe relative to CZTSe. These results indicate the ability for Ag to modify the defect properties of kesterite absorbers, even with only moderate alloying levels. The difference between the carrier density estimated from CV and DLCP suggests the presence of interface or deep-level defects [43], 
though these effects are minimal for the characterized absorbers. Capacitance spectroscopy data for 50\%-ACZTSe is not presented as further information regarding the dielectric constant for this material is necessary to accurately estimate the free carrier density. The characterized increase in minority carrier lifetime for ACZTSe relative to CZTSe is attributed here to the increase in average grain size (i.e. reduced grain boundaries) as well as reduction in defect density for the ACZTSe absorbers.

To characterize photogenerated carrier absorption/collection, EQE analysis was considered for the CZTSe and ACZTSe devices, presented in Fig. 5(a). First, the 50\%-ACZTSe alloy shows a notable increase in $E_{G}$ relative to CZTSe, demonstrating the expected shift in band edges upon Ag-incorporation previously discussed. However, relative to CZTSe, 50\%-ACZTSe also demonstrates an overall reduction in QE data at all wavelengths below $E_{G}$ by c. 30\%; see supporting information for a comparison of the normalized EQE data. A wavelength-independent reduction in the relative QE data is typically associated with a loss mechanism which affects all carriers equally, such as interface recombination, as opposed to diffusion limitations [44]. As an unfavorable spike alignment of the conduction bands at the heterojunction interface for the 50\%-ACZTSe device is expected - as similarly characterized for the analogous ACIGSe material system where Ag-incorporation lowers the CBM due to anion displacement [13] - the reduced EQE data in addition to significant light-dark JV crossover, forward bias JV rollover, and low open-circuit voltage [45] (see supporting information) suggest interface recombination limitations due to an unfavorable conduction band offset. In contrast, CZTSe and the 5\%-ACZTSe device demonstrate similar $\mathrm{EQE}$ behavior, though a slight reduction in the data at long wavelengths is identified for the 5\%-ACZTSe absorber; this reduction is associated with a slight increase in the absorber band gap as well as a reduction in band-tailing (discussed below) for the ACZTSe absorber.
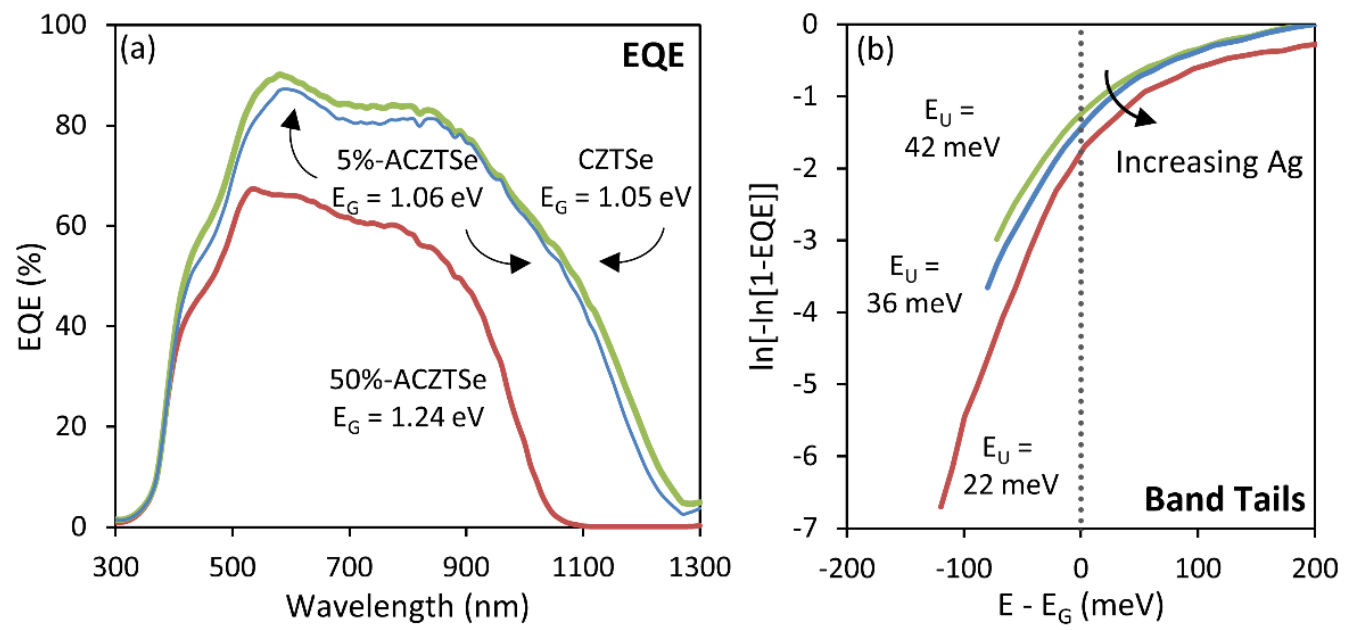

Fig. 5. (a) EQE data for representative CZTSe (thick), 5\%-ACZTSe (thin), and 50\%-ACZTSe devices. (b) $\ln [-\ln [1$ $E Q E]]$ vs. $E-E_{G}$ for Urbach tail analysis, calculated from the $\mathrm{EQE}$ data of (a).

The existence of potential fluctuations/band-tailing in CZTSSe devices has recently been reported as a limiting factor for improved device performance for this material $[2,46]$. To characterize band-tailing for CZTSe and ACZTSe absorbers reported here, an Urbach tail model was applied to account for sub-band gap photon absorption. Under this Urbach tail model, a plot of $\ln [-\ln [1-E Q E]]$ vs. $E$ can be used to extract the characteristic Urbach tail energy $\left(E_{U}\right)$, as described by Travano et al. [47]; the slope of the linear region below $E_{G}$ for the data yields an estimate of $E_{U}$. Band-tail analysis for the CZTSe and ACZTSe absorbers is presented in Fig. 5(b), normalized to the respective absorber band gaps for the materials. Here we see all three absorbers demonstrate sub-band gap photon absorption. Furthermore, a reduction in $E_{U}$ is characterized for ACZTSe with increasing $\mathrm{Ag}$ content relative to CZTSe, with $E_{U}$ for the 50\%-ACZTSe close to that reported for CIGSe devices with notably higher device performance [14]. A reduction in band-tailing with Ag alloying has been similarly reported for Ag-alloyed ACIGSe absorbers relative to CIGSe [14]. These results further demonstrate promise for reduced defect formation of kesterite absorbers with Ag-alloying. It should also be noted that band tail values for CZTSe are reduced relative to that 
previously reported for nanocrystal-based CZTSSe absorbers [2], which we attribute to a reduction in S in the crystal lattice following RTP processing.

Lastly, JV data for the CZTSe and ACZTSe devices is presented in Table 1; see supporting information for JV curves. Here we find that the improved grain growth, increased minority carrier lifetime, reduced free carrier density/defect formation, and reduced potential fluctuations/band-tailing result in improved average $V_{O C}$ and average device performance for the 5\%-ACZTSe device relative to CZTSe with similar band gap. In contrast, 50\%-ACZTSe demonstrates relatively poor device performance (namely $V_{O C}$ ), which we attribute to poor $p n$-heterojunction band alignment (previously discussed); optimization of the heterojunction band alignment, through $n$-type materials such as $\mathrm{Zn}(\mathrm{O}, \mathrm{S})$, may result in improved device performance for the 50\%-ACZTSe alloy, as this material demonstrates enhanced optoelectronic properties related to band tailing and carrier lifetime relative to CZTSe and 5\%-ACZTSe.

Table 1. A summary of average (champion) device performance and extracted optoelectronic properties discussed herein for CZTSe, 5\%-ACZTSe, and 50\%-ACZTSe.

\begin{tabular}{|c|c|c|c|c|c|c|c|}
\hline$\% \mathrm{Ag}$ & $\mathrm{V}_{\mathrm{Oc}}(\mathrm{V})$ & $\mathrm{J}_{\mathrm{SC}}\left(\mathrm{mA} / \mathrm{cm}^{2}\right)$ & $\mathrm{FF}(\%)$ & $\eta(\%)$ & $\mathrm{E}_{\mathrm{G}}(\mathrm{eV})$ & $\mathrm{E}_{\mathrm{U}}(\mathrm{meV})$ & $\tau(\mathrm{ns})$ \\
\hline 0 & $\begin{array}{c}0.35 \pm .006 \\
(0.36)\end{array}$ & $\begin{array}{c}31.6 \pm 1.25 \\
(32.9)\end{array}$ & $\begin{array}{c}59.4 \pm 2.16 \\
(60.5)\end{array}$ & $\begin{array}{c}6.6 \pm 0.31 \\
(7.2)\end{array}$ & 1.05 & 42 & 2.4 \\
\hline 5 & $\begin{array}{c}0.38 \pm .005 \\
(0.37)\end{array}$ & $\begin{array}{c}31.6 \pm 0.73 \\
(31.9)\end{array}$ & $\begin{array}{c}58.8 \pm 1.44 \\
(61.4)\end{array}$ & $\begin{array}{c}7.1 \pm 0.11 \\
(7.2)\end{array}$ & 1.06 & 35 & 3.6 \\
\hline 50 & $\begin{array}{c}\mathbf{0 . 2 8} \pm .009 \\
(0.30)\end{array}$ & $\begin{array}{c}24.1 \pm 0.49 \\
(24.7)\end{array}$ & $\begin{array}{c}44.5 \pm 1.44 \\
(44.4)\end{array}$ & $\begin{array}{c}3.0 \pm 0.19 \\
(3.3)\end{array}$ & 1.24 & 22 & 10.0 \\
\hline
\end{tabular}

Device performance for CZTSe reported here is lower than that previously reported for nanocrystal-based CZTSSe absorbers [3,19]. However, this difference is attributed to the described RTP processing for the sulfur-free absorbers, as the CZTSe absorbers reported here are characterized with a $50 \mathrm{meV}$ reduction in band gap relative to that previously reported for CZTSSe; this reduction in band gap correlates with the characterized reduction in $V_{O C}$, resulting in similar $E_{G} / q-V_{O C}$ values for the CZTSe and CZTSSe devices. Additionally, $\mathrm{MgF}_{2}$ anti-reflective coatings were not utilized for the absorbers reported herein.

\section{Conclusions}

CZTSe and ACZTSe solar cells with 5 at. $\%$ and 50 at. \% nominal Ag-alloying have been fabricated from heterogeneous CZTS and $\mathrm{SnS}-\mathrm{Ag}_{2} \mathrm{~S}$ nanoparticle inks. RTP processing has been used for large-grain formation to achieve fully selenized absorbers. The benefits of Ag-incorporation in the kesterite absorbers have been characterized and include enhanced grain-growth, improved minority carrier lifetime, reduced defect formation/carrier density, and reduced potential fluctuations/band-tailing. These benefits are shown to scale with an increasing degree of Ag-alloying in ACZTSe. Additionally, ACZTSe absorbers have been characterized with a tunable band gap, as similarly found for $\mathrm{S} / \mathrm{Se}$ and $\mathrm{Sn} / \mathrm{Ge}$ alloying. For similar band gap absorbers, improved average device performance is reported for 5\%-ACZTSe relative to CZTSe, which manifests through increased $V_{O C}$. Poor device performance is measured for 50\%-ACZTSe, despite the improved optoelectronic properties of 50\%-ACZTSe relative to 5\%-ACZTSe and CZTSe, which we postulate is attributed to heterojunction band alignment limitations. These initial results indicate the need for further nanocrystal, RTP, and buffer/window layer optimization for ACZTSe solar cells. In conclusion, Ag-alloying demonstrates a promising route to achieve improved device performance for the kesterite material system as Ag-alloying is shown to improve many of the optoelectronic properties recently characterized to limit device performance in CZTSe.

Acknowledgments

R.A gratefully acknowledges the financial support from NSF IGERT 0903670-DGE and DOE SunShot DEEE0005328. 


\section{References}

[1] W. Wang, M.T. Winkler, O. Gunawan, T. Gokmen, T.K. Todorov, Y. Zhu, et al., Device Characteristics of CZTSSe Thin-Film Solar Cells with 12.6\% Efficiency, Adv. Energy Mater. (2013). doi:10.1002/aenm.201301465.

[2] C.J. Hages, N.J. Carter, R. Agrawal, T. Unold, Generalized current-voltage analysis and efficiency limitations in non-ideal solar cells: Case of $\mathrm{Cu} 2 \mathrm{ZnSn}(\mathrm{SxSe} 1-\mathrm{x}) 4$ and $\mathrm{Cu} 2 \mathrm{Zn}(\mathrm{SnyGe} 1-\mathrm{y})(\mathrm{SxSe} 1-\mathrm{x}) 4, \mathrm{~J}$. Appl. Phys. 115 (2014) 234504. doi:10.1063/1.4882119.

[3] C.J. Hages, S. Levcenco, C.K. Miskin, J.H. Alsmeier, D. Abou-Ras, R.G. Wilks, et al., Improved performance of Ge-alloyed CZTGeSSe thin-film solar cells through control of elemental losses, Prog. Photovoltaics Res. Appl. 23 (2015) 376-384. doi:10.1002/pip.2442.

[4] S. Chen, J.-H. Yang, X.G. Gong, A. Walsh, S.-H. Wei, Intrinsic point defects and complexes in the quaternary kesterite semiconductor Cu2ZnSnS4, Phys. Rev. B. 81 (2010) 245204. doi:10.1103/PhysRevB.81.245204.

[5] S. Levcenko, V.E. Tezlevan, E. Arushanov, S. Schorr, T. Unold, Free-to-bound recombination in near stoichiometric Cu2ZnSnS4 single crystals, Phys. Rev. B. 86 (2012) 45206.

doi:10.1103/PhysRevB.86.045206.

[6] T. Gershon, B. Shin, N. Bojarczuk, T. Gokmen, S. Lu, S. Guha, Photoluminescence characterization of a high-efficiency Cu2ZnSnS4 device, J. Appl. Phys. 114 (2013) 2011-2016. doi:10.1063/1.4825317.

[7] M.J. Romero, H. Du, G. Teeter, Y. Yan, M.M. Al-Jassim, Comparative study of the luminescence and intrinsic point defects in the kesterite $\mathrm{Cu} 2 \mathrm{ZnSnS} 4$ and chalcopyrite $\mathrm{Cu}(\mathrm{In}, \mathrm{Ga}) \mathrm{Se} 2$ thin films used in photovoltaic applications, Phys. Rev. B. 84 (2011) 165324. doi:10.1103/PhysRevB.84.165324.

[8] S. Chen, A. Walsh, J.-H. Yang, X.G. Gong, L. Sun, P.-X. Yang, et al., Compositional dependence of structural and electronic properties of $\mathrm{Cu} 2 \mathrm{ZnSn}(\mathrm{S}, \mathrm{Se}) 4$ alloys for thin film solar cells, Phys. Rev. B. 83 (2011) 125201. doi:10.1103/PhysRevB.83.125201.

[9] Q. Guo, G.M. Ford, W.-C. Yang, C.J. Hages, H.W. Hillhouse, R. Agrawal, Enhancing the performance of CZTSSe solar cells with Ge alloying, Sol. Energy Mater. Sol. Cells. 105 (2012) 132-136.

doi:10.1016/j.solmat.2012.05.039.

[10] S. Chen, X. Gong, A. Walsh, S.-H. Wei, Electronic structure and stability of quaternary chalcogenide semiconductors derived from cation cross-substitution of II-VI and I-III-VI2 compounds, Phys. Rev. B. 79 (2009) 165211. doi:10.1103/PhysRevB.79.165211.

[11] R. Scheer, H.-W. Schock, Design Rules for Heterostructure Solar Cells and Modules, in: Chalcogenide Photovoltaics Physics, Technol. Thin Film Devices, Wiley-VCH Verlag GmbH \& Co. KGaA, Weinheim, Germany, 2011: pp. 129-174. doi:10.1002/9783527633708.

[12] J.H. Boyle, B.E. McCandless, W.N. Shafarman, R.W. Birkmire, Structural and optical properties of $(\mathrm{Ag}, \mathrm{Cu})(\mathrm{In}, \mathrm{Ga}) \mathrm{Se} 2$ polycrystalline thin film alloys, J. Appl. Phys. 115 (2014) 0-8. doi:10.1063/1.4880243.

[13] S. Chen, X.G. Gong, S.H. Wei, Band-structure anomalies of the chalcopyrite semiconductors CuGa X2 versus AgGa X2 (X=S and Se) and their alloys, Phys. Rev. B - Condens. Matter Mater. Phys. 75 (2007) 1-9. doi:10.1103/PhysRevB.75.205209. 
[14] P.T. Erslev, J. Lee, G.M. Hanket, W.N. Shafarman, J.D. Cohen, The electronic structure of $\mathrm{Cu}(\mathrm{In} 1-\mathrm{xGax}) \mathrm{Se} 2$ alloyed with silver, Thin Solid Films. 519 (2011) 7296-7299. doi:10.1016/j.tsf.2011.01.368.

[15] W. Li, X. Liu, H. Cui, S. Huang, X. Hao, The role of Ag in (Ag,Cu)2ZnSnS4 thin film for solar cell application, J. Alloys Compd. 625 (2015) 277-283. doi:10.1016/j.jallcom.2014.11.136.

[16] W. Gong, T. Tabata, K. Takei, M. Morihama, T. Maeda, T. Wada, Crystallographic and optical properties of (Cu,Ag)2ZnSnS4 and (Cu,Ag)2ZnSnSe4 solid solutions, Phys. Status Solidi. 4 (2015) 1-4. doi:10.1002/pssc.201400343.

[17] K. Wei, G.S. Nolas, Synthesis and Characterization of Nanostructured Stannite Cu2ZnSnSe4 and Ag2ZnSnSe4 for Thermoelectric Applications, ACS Appl. Mater. Interfaces. 7 (2015) 9752-9757. doi:10.1021/acsami.5b01617.

[18] T. Sasamura, T. Osaki, T. Kameyama, T. Shibayama, A. Kudo, S. Kuwabata, et al., Solution-phase Synthesis of Stannite-type Ag2ZnSnS4 Nanoparticles for Application to Photoelectrode Materials, Chem. Lett. 41 (2012) 1009-1011. doi:10.1246/cl.2012.1009.

[19] C.K. Miskin, W.-C. Yang, C.J. Hages, N.J. Carter, C.S. Joglekar, E.A. Stach, et al., 9.0\% efficient $\mathrm{Cu} 2 \mathrm{ZnSn}(\mathrm{S}, \mathrm{Se}) 4$ solar cells from selenized nanoparticle inks, Prog. Photovoltaics Res. Appl. 23 (2015) 654-659. doi:10.1002/pip.2472.

[20] C.J. Hages, R. Agrawal, Synthesis of CZTSSe Thin Films from Nanocrystal Inks, in: K. Ito (Ed.), Copp. Zinc Tin Sulfide-Based Thin Film Sol. Cells, 1st ed., John Wiley \& Sons, Ltd., West Sussex, UK, 2015: pp. 239-270.

[21] G. Zoppi, I. Forbes, R.W. Miles, P.J. Dale, J.J. Scragg, L.M. Peter, Cu2ZnSnSe4 Thin Film Solar Cells Produced by Selenisation of Magnetron Sputtered Precursors, Prog. Photovoltaics Res. Appl. 17 (2009) 315-319. doi:10.1002/pip.

[22] J.L. Pankove, Absorption, in: Opt. Process. Semicond., Dover Publications, New York, 1971: pp. 34-86.

[23] J.T. Heath, J.D. Cohen, W.N. Shafarman, Bulk and metastable defects in CuIn1-xGaxSe2 thin films using drive-level capacitance profiling, J. Appl. Phys. 95 (2004) 1000. doi:10.1063/1.1633982.

[24] C.J. Hages, N.J. Carter, J. Moore, S.M. McLeod, C.K. Miskin, C. Joglekar, et al., Device comparison of champion nanocrystal-ink based CZTSSe and CIGSSe solar cells: Capacitance spectroscopy, in: IEEE 39th Photovolt. Spec. Conf., IEEE, Tampa, FL, 2013: pp. 1966-1971. doi:10.1109/PVSC.2013.6744856.

[25] W.K. Metzger, I. Repins, M. Romero, P. Dippo, M.A. Contreras, R. Noufi, et al., Recombination kinetics and stability in polycrystalline $\mathrm{Cu}(\mathrm{In}, \mathrm{Ga}) \mathrm{Se} 2$ solar cells, Thin Solid Films. 517 (2009) 2360-2364.

[26] M. Grossberg, J. Krustok, J. Raudoja, K. Timmo, M. Altosaar, T. Raadik, Photoluminescence and Raman study of Cu2ZnSn(SexS1-x)4 monograins for photovoltaic applications, Thin Solid Films. 519 (2011) 7403-7406. doi:10.1016/j.tsf.2010.12.099.

[27] N.J. Carter, W.C. Yang, C.K. Miskin, C.J. Hages, E. a. Stach, R. Agrawal, Cu2ZnSn(S,Se)4 solar cells from inks of heterogeneous Cu-Zn-Sn-S nanocrystals, Sol. Energy Mater. Sol. Cells. 123 (2014) 189-196. doi:10.1016/j.solmat.2014.01.016.

[28] A.R. Denton, N.W. Ashcroft, Vegards law, Phys. Rev. A. 43 (1991) 3161-3164. doi:10.1103/PhysRevA.43.3161. 
[29] R.H. Eric, H. Ozok, High-temperature phase relations and thermodynamics in the silver-tin-sulfur system, Metall. Mater. Trans. B. 30B (1999) 707-714.

[30] H.R. Chandrasekhar, R.G. Humphreys, U. Zwick, M. Cardona, Infrared and Raman spectra of the IV-VI compounds SnS and SnSe, Phys. Rev. B. 15 (1977) 2177-2183. doi:10.1103/PhysRevB.15.2177.

[31] B. Minceva-Sukarova, M. Najdoski, I. Grozdanov, C.J.J. Chunnilall, Raman spectra of thin solid films of some metal sulfides, J. Mol. Struct. 410-411 (1997) 267-270. doi:10.1016/S0022-2860(96)09713-X.

[32] M. Guc, S. Levcenco, V. Izquierdo-Roca, X. Fontané, E. Arushanov, A. Perez-Rodriguez, A polarized Raman scattering analysis of Cu2ZnSnSe4 and Cu2ZnGeSe4 single crystals, Jpn. J. Appl. Phys. 114 (2013) 193514-1 - 193514-9. doi:10.1063/1.4830028.

[33] A. Redinger, S. Siebentritt, Loss Mechanisms in Kesterite Solar Cells, in: K. Ito (Ed.), Copp. Zinc Tin Sulfide-Based Thin Film Sol. Cells, 1st ed., John Wiley \& Sons, Ltd., West Sussex, UK, 2015: pp. 363-386.

[34] Y. Cao, M.S. Denny Jr., J. V Caspar, W.E. Farneth, Q. Guo, A.S. Ionkin, et al., High-Efficiency SolutionProcessed Cu2ZnSn(S,Se)4 Thin-Film Solar Cells Prepared from Binary and Ternary Nanoparticles, J. Am. Chem. Soc. 134 (2012) 15644-7. doi:10.1021/ja3057985.

[35] H. Cui, X. Liu, F. Liu, X. Hao, N. Song, C. Yan, Boosting Cu2ZnSnS4 solar cells efficiency by a thin Ag intermediate layer between absorber and back contact, Appl. Phys. Lett. 104 (2014) 041115. doi:10.1063/1.4863951.

[36] G. Rey, A. Redinger, J. Sendler, T.P. Weiss, M. Thevenin, M. Guennou, et al., The band gap of Cu2ZnSnSe4: Effect of order-disorder, Appl. Phys. Lett. 112106 (2014). doi:10.1063/1.4896315.

[37] C.M. Sutter-Fella, J. a. Stückelberger, H. Hagendorfer, F. La Mattina, L. Kranz, S. Nishiwaki, et al., Sodium Assisted Sintering of Chalcogenides and Its Application to Solution Processed Cu2ZnSn(S,Se)4 Thin Film Solar Cells, Chem. Mater. 26 (2014) 1420-1425. doi:10.1021/cm403504u.

[38] H. Guo, Y. Cui, Q. Tian, S. Gao, G. Wang, D. Pan, Significantly Enhancing Grain Growth in $\mathrm{Cu} 2 \mathrm{ZnSn}(\mathrm{S}, \mathrm{Se}) 4$ Absorber Layers by Insetting Sb2S3, CuSbS2, and NaSb5S8 Thin Films, Cryst. Growth Des. 15 (2015) 771-777. doi:10.1021/cg501589t.

[39] W.C. Hsu, I. Repins, C. Beall, G. Teeter, C. Dehart, B. To, et al., Growth kinetics during kesterite coevaporation, Proc. 38th IEEE Photovolt. Spec. Conf. (2012) 674-677. doi:10.1109/PVSC.2012.6317699.

[40] R. Klenk, T. Walter, H.-W. Schock, D. Cahen, A model for the successful growth of polycrystalline films of CuInSe2 by multisource physical vacuum evaporation, Adv. Mater. 5 (1993) 114-119. doi:10.1002/adma.19930050209.

[41] T. Gershon, B. Shin, N. Bojarczuk, M. Hopstaken, D.B. Mitzi, S. Guha, The role of sodium as a surfactant and suppressor of non-radiative recombination at internal surfaces in $\mathrm{Cu} 2 \mathrm{ZnSnS4}$, Adv. Energy Mater. (2014) 1-8. doi:10.1002/aenm.201400849.

[42] I.L. Repins, H. Moutinho, S.G. Choi, a. Kanevce, D. Kuciauskas, P. Dippo, et al., Indications of short minority-carrier lifetime in kesterite solar cells, J. Appl. Phys. 114 (2013) 084507. doi:10.1063/1.4819849.

[43] J.R. Heath, P. Zabierowski, Capacitance Spectroscopy of Thin-Film Solar Cells, in: D. Abou-Ras, T. Kirchartz, U. Rau (Eds.), Adv. Charact. Tech. Thin Film Sol. Cells, WILEY_VCH Verlag Gmbh \& Co. KGaA, Weinheim, Germany, 2011: pp. 81-105. 
[44] S.S. Hegedus, W.N. Shafarman, Thin-film solar cells: device measurements and analysis, Prog. Photovoltaics Res. Appl. 12 (2004) 155-176. doi:10.1002/pip.518.

[45] R. Scheer, H.W. Schock, Appendix A: Frequently Observed Anomalies, in: Chalcogenide Photovoltaics Physics, Technol. Thin Film Devices, Wiley-VCH Verlag GmbH \& Co. KGaA, Weinheim, Germany, 2011: pp. 305-314. doi:10.1002/9783527633708.

[46] T. Gokmen, O. Gunawan, T.K. Todorov, D.B. Mitzi, Band tailing and efficiency limitation in kesterite solar cells, Appl. Phys. Lett. 103 (2013) 103506. doi:10.1063/1.4820250.

[47] M. Troviano, K. Taretto, Analysis of internal quantum efficiency in double-graded bandgap solar cells including sub-bandgap absorption, Sol. Energy Mater. Sol. Cells. 95 (2011) 821-828. doi:10.1016/j.solmat.2010.10.028. 
